\title{
Síndrome de Heyde: Estratégias Terapêuticas e Seguimento de Longo Prazo
}

\author{
Heyde's Syndrome: Therapeutic Strategies and Long-Term Follow-Up
}

Vitor Emer Egypto Rosa, ${ }^{1}$ Henrique Barbosa Ribeiro, ${ }^{\circledR}$ João Ricardo Cordeiro Fernandes, ${ }^{1}$ Antonio de Santis, ${ }^{1}$ Guilherme Sobreira Spina, ${ }^{1}$ Milena Ribeiro Paixão, ${ }^{1}$ Lucas José Tachotti Pires, ${ }^{1}$ Marcelo Bettega, ${ }^{10}$ Tarso Augusto Duenhas Accorsi, ${ }^{1}$ Roney Orismar Sampaio, ${ }^{1}$ Flávio Tarasoutchi ${ }^{1}$

Instituto do Coração do Hospital das Clinicas da Faculdade de Medicina da Universidade de São Paulo, ${ }^{1}$ São Paulo, SP - Brasil

\section{Resumo}

Fundamentos: A síndrome de Heyde é a associação de estenose aórtica importante com episódio de sangramento gastrointestinal por lesões angiodisplásicas. Pouco é conhecido sobre os fatores associados a novos sangramentos e desfechos em longo prazo. Além disso, a maioria dos dados é restrita a relatos de casos e pequenas séries.

Objetivo: Avaliar o perfil clínico, laboratorial e ecocardiográfico de pacientes com síndrome de Heyde submetidos a intervenção valvar ou tratamento medicamentoso.

Métodos: Coorte prospectiva de 24 pacientes consecutivos entre 2005 e 2018 . Foram avaliados dados clínicos, laboratoriais, ecocardiográficos e relacionados à intervenção valvar e a desfechos após o diagnóstico. Valor de p<0,05 foi considerado estatisticamente significante.

Resultados: Metade dos 24 pacientes apresentou sangramento com necessidade de transfusão sanguínea na admissão. Angiodisplasias foram encontradas mais frequentemente no cólon ascendente (62\%). Intervenção valvar (cirúrgica ou transcateter) foi realizada em $70,8 \%$ dos pacientes, e $29,2 \%$ foram mantidos em tratamento clínico. Novos episódios de sangramento ocorreram em $25 \%$ dos casos, e não houve diferença entre os grupos clínico e intervenção (28,6 vs. $23,5 \%, p=1,00$; respectivamente). A mortalidade no seguimento de 2 e 5 anos foi de $16 \%$ e $25 \%$, sem diferença entre os grupos (log-rank $p=0,185$ e 0,737 , respectivamente).

Conclusões: Pacientes com síndrome de Heyde tiveram alta taxa de sangramento com necessidade de transfusão sanguínea na admissão, sugerindo ser uma doença grave e com risco elevado de mortalidade. Não encontramos diferenças entre os grupos submetidos ao tratamento clínico e à intervenção valvar em relação a taxas de ressangramento e mortalidade tardia.

Palavras-chave: Estenose Aórtica; Angiodisplasia; Hemorragia; Mortalidade; Cirurgia Torácica; Ecocardiografia/métodos.

\section{Abstract}

Background: Heyde's syndrome is the association of severe aortic stenosis with episodes of gastrointestinal due to angiodysplastic lesion. Little is known about the factors associated with new episodes of bleeding and long-term outcomes. Furthermore, most data are restricted to case reports and small case series.

Objective: To assess the clinical, laboratory and echocardiography profile of patients with Heyde's syndrome who underwent valve intervention or drug therapy.

Methods: Prospective cohort of 24 consecutive patients from 2005 to 2018. Clinical, laboratory and echocardiography data were assessed, as well as those related to valve intervention and to outcomes after diagnosis. Statistical significance was set $p<0.05$.

Results: Half of the 24 patients (50\%) presented with bleeding requiring blood transfusion on admission. Angiodysplasias were more frequently found in the ascending colon (62\%). Valve intervention (surgical or transcatheter) was performed in $70.8 \%$ of the patients, and $29.2 \%$ remained on drug therapy. News episodes of bleeding occurred in $25 \%$ of the cases, and there was no difference between clinical and intervention groups (28.6 vs 23.5\%, $p=1.00$; respectively). Mortality at 2-year and 5-year follow-up was 16\% and 25\%, with no difference between the groups (log-rank $p=0.185$ and 0.737 , respectively).

Conclusions: Patients with Heyde's syndrome had a high rate of bleeding requiring blood transfusion on admission, suggesting that is a severe disease with high mortality risk. No difference was found between the groups subjected to clinical treatment and to valve intervention with regard to rates of rebleeding and late mortality.

Keywords: Aortic Stenosis; Angiodysplasia; Hemorrhage; Mortality; Thoracic Surgery; Echocardiography/methods.

Full texts in English - http://www.arquivosonline.com.br

Correspondência: Vitor Emer Egypto Rosa •

Instituto do Coração do Hospital das Clinicas da Faculdade de Medicina da Universidade de São Paulo - Dr. Eneras Carvalho de Aguiar, 44. CEP 05403-000, São Paulo, SP - Brasil

E-mail: vitoremer@yahoo.com.br

Artigo recebido em 08/05/2020, revisado em 16/08/2020, aceito em 09/09/2020

DOI: https://doi.org/10.36660/abc.20200371 


\section{Introdução}

A associação entre sangramento gastrointestinal por lesões angiodisplásicas e estenose aórtica importante é denominada síndrome de Heyde e foi descrita por Edward Heyde em $1958 .{ }^{1}$ Desde então, ainda há controvérsias sobre a patogênese de tal síndrome, e o único fator que comprovadamente predispõe a sangramentos nessa população é a presença da deficiência adquirida do fator de von Willebrand (von Willebrand factor, vWF) decorrente do estresse hemodinâmico no orifício valvar aórtico estenótico. ${ }^{2-6}$

A síndrome de Heyde é descrita em 1,7\% dos pacientes com estenose aórtica importante e, apesar de sua prevalência, pouco é conhecido sobre seus aspectos epidemiológicos, e a maioria das publicações relacionadas a essa entidade são relatos de casos e pequenas séries. ${ }^{5} \mathrm{~A}$ indicação de intervenção valvar nesses pacientes segue as diretrizes atuais e, apesar de não existirem recomendações específicas, alguns centros advogam intervenção para redução de sangramento independente da presença de sintomas ou complicadores. ${ }^{7-9}$ Assim, o objetivo deste estudo foi avaliar os aspectos clínicos, laboratoriais e ecocardiográficos, além da mortalidade e de complicações após intervenção valvar ou tratamento clínico, em uma série de casos de pacientes com síndrome de Heyde.

\section{Métodos}

Trata-se de uma coorte prospectiva que avaliou pacientes com estenose aórtica importante em um centro terciário entre 2005 e 2018. A estenose aórtica importante foi definida como a presença de área valvar aórtica $\leq 1,0 \mathrm{~cm}^{2}$, gradiente transaórtico médio $>40 \mathrm{mmHg}$ ou velocidade de pico $>4,0 \mathrm{~m} / \mathrm{s}$ pelo ecocardiograma transtorácico. ${ }^{9}$ Pacientes com história de sangramento gastrointestinal e angiodisplasia documentada por colonoscopia e/ou endoscopia digestiva alta foram selecionados. Foram avaliados dados clínicos, laboratoriais, ecocardiográficos e relacionados ao tratamento indicado. Os critérios de exclusão foram: presença de outras condições que pudessem justificar o sangramento (como úlcera gástrica ou neoplasias), insuficiência aórtica importante ou valvopatia mitral primária anatomicamente importante. A indicação da intervenção valvar foi realizada de acordo com a escolha do médico assistente e seguindo as diretrizes vigentes. ${ }^{9,10}$ Para fins comparativos, dividimos os pacientes em dois grupos, de acordo com o tratamento escolhido (intervenção valvar vs. tratamento clínico medicamentoso). Avaliamos mortalidade e complicações perioperatórias em 30 dias e, após, através de contato telefônico. Este estudo foi aprovado pelo comitê de ética e pesquisa institucional, tendo sido solicitada a dispensa do termo de consentimento.

\section{Análise estatística}

As variáveis contínuas e categóricas foram apresentadas como mediana (intervalo interquartil) e frequências ou porcentagens, respectivamente. A normalidade das variáveis foi avaliada com o teste de Kolmogorov-Smirnov. Variáveis contínuas foram analisadas através do teste de Mann-Whitney e variáveis categóricas através teste chi-quadrado ou teste exato de Fisher, conforme apropriado. A mortalidade em 5 anos foi avaliada utilizando teste Log-rank. Todos os testes foram bicaudais, e um valor de $p<0,05$ foi utilizado para indicar significância estatística. O software SPSS versão 20 (IBM, Armonk, New York, USA) foi utilizado para analisar os dados.

\section{Resultados}

Um total de 24 pacientes apresentou perfil para inclusão no estudo. As características basais da população estão descritas na Tabela 1. A mediana etária foi de 77 [70-82] anos, $50 \%$ dos pacientes eram do sexo feminino, e encontramos uma alta prevalência de comorbidades como hipertensão arterial sistêmica $(79,2 \%)$, diabetes $(41,7 \%)$ e doença arterial coronariana $(41,7 \%)$. Todos os pacientes apresentaram sangramento gastrointestinal, porém 50\% receberam transfusão de concentrados de hemáceas na admissão. Apenas $33,3 \%$ faziam uso de antiagregantes plaquetários (aspirina), e $8,3 \%$ de anticoagulação com varfarina. Não houve diferença em relação aos sangramentos com necessidade de transfusão comparando os pacientes em uso de aspirina vs. aqueles sem essa medicação (62,5 vs. 43,8\%, respectivamente; $p=0,385)$, assim como em relação ao uso de varfarina, já que os únicos dois pacientes em uso dessa medicação não apresentaram novos sangramentos.

Todos os pacientes eram portadores de estenose aórtica de etiologia degenerativa, com mediana de gradiente transaórtico médio de 49 [42-57] $\mathrm{mmHg}$, área valvar aórtica de 0,66 [0,60-0,70] $\mathrm{cm}^{2}$ e velocidade de pico de $4,5[4,0-4,9] \mathrm{m} / \mathrm{s}$. A mediana da fração de ejeção do ventrículo esquerdo foi de 64 [56-68] \%. O restante das características ecocardiográficas está descrito na Tabela 1, assim como os dados laboratoriais. A hemoglobina basal era de $10,1[8,2-11,4] \mathrm{g} / \mathrm{dL}$, e $17,4 \%$ e $34,8 \%$ dos pacientes apresentavam hemoglobina $<7,0$ e $<9,0 \mathrm{mg} / \mathrm{dL}$ na admissão, respectivamente. Os valores do coagulograma eram normais, com exceção de um paciente em anticoagulação oral com razão normalizada internacional $(\mathrm{RNI})$ de 1,7. Em relação à colonoscopia e à endoscopia digestiva alta, os dados basais estão descritos na Tabela 2. Todos os pacientes apresentavam angiodisplasia, 50,0\% diagnosticada na colonoscopia, $33,3 \%$ na endoscopia digestiva alta, e 16,6\% em ambas. As lesões foram mais frequentemente encontradas no cólon ascendente $(62,5 \%)$ e no estômago (37,5\%). Tratamento das lesões com cauterização ou argônio ocorreu em 41,7\% dos casos.

\section{Intervenção valvar vs. tratamento clínico}

Os dados relacionados à intervenção e a eventos estão descritos na Tabela 3. Em nosso estudo, sete (29,2\%) pacientes foram mantidos em tratamento clínico, e 17 (70,8\%) foram submetidos à intervenção valvar aórtica, sendo que em $70,5 \%$ foi implantada prótese biológica, em 17,6\%; prótese mecânica; em 11,7\%, implante transcateter de bioprótese aórtica (TAVR); e, em 23,5\%, foi realizada revascularização miocárdica concomitante. A mortalidade em 30 dias e 1 ano do grupo intervenção foi de 11,8\% e 23,5\%, respectivamente. Não encontramos diferenças relacionadas às características basais, laboratoriais, ecocardiográficas nem relacionadas à endoscopia digestiva alta e colonoscopia na comparação 
Tabela 1 - Características clínicas, laboratoriais e ecocardiográficas basais da população estudada

\begin{tabular}{|c|c|c|c|c|}
\hline & Total $(n=24)$ & Intervenção (n=17) & Clínico $(n=7)$ & p \\
\hline \multicolumn{5}{|l|}{ Características clínicas } \\
\hline Idade (anos) & $77[70-82]$ & $76[68-81]$ & $82[81-87]$ & 0,069 \\
\hline Sexo feminino, $\mathrm{n}(\%)$ & $12(50,0)$ & $7(41,2)$ & $5(71,4)$ & 0,371 \\
\hline Diabetes mellitus, $n(\%)$ & $10(41,7)$ & $7(41,2)$ & $3(42,9)$ & 1,000 \\
\hline Hipertensão, n (\%) & $19(79,2)$ & $15(88,2)$ & $4(57,1)$ & 0,126 \\
\hline Fibrilação atrial, n (\%) & $4(16,7)$ & $3(17,6)$ & $1(14,3)$ & 1,000 \\
\hline Doença arterial coronariana, n (\%) & $10(41,7)$ & $8(47,1)$ & $2(8,6)$ & 0,653 \\
\hline EuroSCORE II (\%) & $1,78[1,44-3,42]$ & $1,71[1,31-2,81]$ & $2,41[1,57-7,55]$ & 0,371 \\
\hline STS $(\%)$ & $2,43[1,60-4,42]$ & $2,21[1,49-3,95]$ & $4,8[1,93-6,04]$ & 1,000 \\
\hline \multicolumn{5}{|l|}{ Sintomas } \\
\hline Dispneia (NYHA III/IV), n (\%) & $14(58,3)$ & $10(58,8)$ & $4(57,1)$ & 1,000 \\
\hline Angina, n (\%) & $3(12,5)$ & $3(17,6)$ & $0(0)$ & 0,530 \\
\hline Sangramento gastrointestinal com necessidade de transfusão, $n(\%)$ & $12(50,0)$ & $9(52,9)$ & $3(42,9)$ & 1,000 \\
\hline \multicolumn{5}{|l|}{ Medicações } \\
\hline Inibidores da ECA ou BRA, n (\%) & $10(41,7)$ & $8(47,1)$ & $2(28,6)$ & 0,653 \\
\hline Betabloqueadores, $\mathrm{n}(\%)$ & $6(25,0)$ & $4(23,5)$ & $2(28,6)$ & 1,000 \\
\hline Antiagregantes plaquetários, n (\%) & $8(33,3)$ & $5(29,4)$ & $3(42,9)$ & 0,647 \\
\hline Diuréticos, n (\%) & $17(70,8)$ & $11(64,7)$ & $6(85,7)$ & 0,625 \\
\hline Estatinas, n (\%) & $9(37,5)$ & $6(35,3)$ & $3(42,9)$ & 1,000 \\
\hline Digoxina, n (\%) & $3(12,5)$ & $2(11,8)$ & $1(14,3)$ & 1,000 \\
\hline Anticoagulação oral, n (\%) & $2(8,3)$ & $2(11,8)$ & $0(0)$ & 1,000 \\
\hline \multicolumn{5}{|l|}{ Parâmetros ecocardiográficos } \\
\hline Gradiente transaórtico médio, $\mathrm{mmHg}$ & 49 [42-57] & $53[42-57]$ & 42 [39-59] & 0,141 \\
\hline Velocidade de pico aórtica, m/s & $4,5[4,0-4,9]$ & $4,5[4,0-5,1]$ & $4,4[4,0-4,8]$ & 0,381 \\
\hline Fração de ejeção do ventrículo esquerdo, \% & $64[56-68]$ & $64[55-68]$ & $65[55-69]$ & \\
\hline Área valvar aórtica, $\mathrm{cm}^{2}$ & $0,66[0,60-0,70]$ & $0,66[0,60-0,70]$ & $0,66[0,60-0,70]$ & 1,000 \\
\hline Insuficiência aórtica moderada, n (\%) & $2(8,3)$ & $1(5,9)$ & $1(14,3)$ & 0,507 \\
\hline Insuficiência mitral funcional moderada/importante, n (\%) & $7(29,2)$ & $5(29,4)$ & $2(28,6)$ & 1,000 \\
\hline Insuficiência tricúspide moderada/importante, n (\%) & $3(12,5)$ & $1(5,9)$ & $2(28,6)$ & 0,194 \\
\hline \multicolumn{5}{|l|}{ Dados laboratoriais } \\
\hline Hemoglobina, g/dL & $10,1[8,2-11,4]$ & $10,4[8,5-12,6]$ & $9,1[6,3-11]$ & 1,000 \\
\hline Hemoglobina < 7,0 g/dL na admissão, n (\%) & $4(17,4)$ & $1(6,3)$ & $3(42,9)$ & 0,067 \\
\hline Hemoglobina < 9,0 g/dL na admissão, n (\%) & $8(34,8)$ & $5(51,3)$ & $3(42,9)$ & 0,657 \\
\hline Hematócrito, \% & $32[25-34]$ & $32[27-36]$ & 27 [22-33] & 0,657 \\
\hline Plaquetas, $/ \mathrm{mm}^{3}$ & 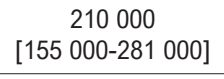 & $\begin{array}{c}208000 \\
\text { [149 000-267 500] }\end{array}$ & $\begin{array}{c}270000 \\
{\left[\begin{array}{lll}175 & 000-299 & 000]\end{array}\right]}\end{array}$ & 0,667 \\
\hline Tempo de protrombina, seg & $15,3[13,2-16,2]$ & $14,6[12,7-16,1]$ & $15,9[15,3-17,9]$ & 0,193 \\
\hline Atividade protrombínica, \% & $78,7[71,8-92,0]$ & $83,6[73,5-92,0]$ & $71,8[63,0-89,8]$ & 0,371 \\
\hline Relação de tempos (TP/TR) & $1,1[1,0-1,2]$ & $1,0[1,0-1,1]$ & $1,2[1,0-1,2]$ & 0,650 \\
\hline RNI & $1,1[1,0-1,2]$ & $1,0[1,0-1,1]$ & $1,2[1,0-1,3]$ & 0,137 \\
\hline TTPA, seg & $29,5[28,2-33,9]$ & $29,3[28,3-31]$, & $30,0[28,1-36,5]$ & 0,361 \\
\hline Clearance de creatinina, $\mathrm{mL} / \mathrm{min} / 1,73 \mathrm{~m}^{2}$ & 59 [48-75] & $61[52-70]$ & 28 [28-77] & 1,000 \\
\hline Ureia, mg/dL & $50[41-70]$ & $47[38-56]$ & $71[50-138]$ & 0,193 \\
\hline
\end{tabular}

BRA: bloqueador do receptor da angiotensina; ECA: enzima conversora da angiotensina; NYHA: New York Heart Association; RNI: razão normalizada internacional; TP: tempo de protrombina; TR: tempo de reptilase; TTPA: tempo de tromboplastina parcial ativada. 


\section{Tabela 2 - Características da colonoscopia e endoscopia digestiva alta}

\begin{tabular}{|c|c|c|c|c|}
\hline & Total $(n=24)$ & Intervenção (n=17) & Clínico $(n=7)$ & $\mathrm{p}$ \\
\hline \multicolumn{5}{|l|}{ Angiodisplasia, $\mathrm{n}(\%)^{*}$} \\
\hline Cólon ascendente, n (\%) & $15(62,5)$ & $9(52,9)$ & $6(85,7)$ & 0,191 \\
\hline Cólon transverso, n (\%) & $2(8,3)$ & $0(0)$ & $2(28,6)$ & 0,076 \\
\hline Estômago, n (\%) & $9(37,5)$ & $7(41,2)$ & $2(28,6)$ & 0,669 \\
\hline Cólon descendente, n (\%) & $4(16,7)$ & $1(5,9)$ & $3(42,9)$ & 0,059 \\
\hline Duodeno, n (\%) & $5(20,8)$ & $4(23,5)$ & $1(14,3)$ & 1,000 \\
\hline Jejuno, n (\%) & $4(16,7)$ & $2(11,8)$ & $2(28,6)$ & 0,552 \\
\hline Divertículos, n (\%) & $13(54,2)$ & $8(47,1)$ & $5(71,4)$ & 0,386 \\
\hline Pólipos, n (\%) & $8(3,3)$ & $7(41,2)$ & $1(14,3)$ & 0,352 \\
\hline Cauterização ou aplicação de argônio, n (\%) & $10(41,7)$ & $9(52,9)$ & $1(14,3)$ & 0,172 \\
\hline
\end{tabular}

${ }^{*} A$ soma pode ser maior que $100 \%$ pois pacientes podem apresentar mais do que uma localidade da angiodisplasia.

Tabela 3 - Características da intervenção e eventos em 30 dias

\begin{tabular}{|c|c|c|c|}
\hline & Intervenção (n=17) & Clínico $(n=7)$ & p \\
\hline Prótese biológica, n (\%) & $12(70,5)$ & - & - \\
\hline Prótese mecânica, n (\%) & $3(17,6)$ & - & - \\
\hline Revascularização miocárdica concomitante, n (\%) & $4(23,5)$ & - & - \\
\hline TAVR, n (\%) & $2(11,7)$ & & \\
\hline Mortalidade em 30 dias, n (\%) & $2(11,8)$ & $0(0)$ & 1,000 \\
\hline Mortalidade em 1 ano, n (\%) & $4(23,5)$ & $0(0)$ & 0,283 \\
\hline Acidente vascular cerebral incapacitante, n (\%) & $1(5,9)$ & - & - \\
\hline Derrame pericárdico, n (\%) & $3(17,6)$ & - & - \\
\hline Fibrilação atrial, n (\%) & $6(35,3)$ & - & - \\
\hline Reabordagem, n (\%) & $2(11,8)$ & - & - \\
\hline Sangramento gastrointestinal, n (\%) & $4(23,5)$ & $2(28,6)$ & 1,000 \\
\hline Sangramento gastrointestinal com necessidade de transfusão, n (\%) & $2(11,8)$ & $2(28,6)$ & 0,552 \\
\hline
\end{tabular}

TAVR indica implante de bioprótese aórtica transcateter, do inglês transcatheter aortic valve replacement.

entre os grupos (Tabelas 1 e 2). Além disso, a taxa de novos sangramentos foi semelhante nos grupos de tratamento clínico vs. intervencionista (28,6 vs. 23,5\%, respectivamente; $p=1,000)$. Dos pacientes do grupo intervenção que apresentaram novos sangramentos gastrointestinais, dois deles necessitaram transfusão de concentrado de hemáceas, e em nenhum desses pacientes a prótese mecânica havia sido implantada. A indicação da intervenção valvar foi presença de sintomas em $15(88,2 \%)$ casos e sangramentos com necessidade de transfusão de concentrados de hemáceas em dois $(11,7 \%)$ casos. Os óbitos em 1 ano foram decorrentes de: endocardite infecciosa em um caso, sepse em um caso, e choque cardiogênico em dois casos.

Três pacientes do grupo intervenção foram submetidos a nova colonoscopia devido a recorrência de sangramento, sendo evidenciada manutenção das lesões angiodisplásicas nas mesmas localidades encontradas antes da intervenção valvar.
A indicação do manejo clínico medicamentoso em sete $(29,2 \%)$ pacientes foi devido à escolha do paciente e/ou do médico assistente em função da idade, da fragilidade clínica e de outras comorbidades. A mediana do seguimento após diagnóstico da síndrome de Heyde foi de 24 [12-54] meses, e a mortalidade global no seguimento de 2 e 5 anos foi de $16 \%$ e $25 \%$, respectivamente, sem diferença entre os grupos (log-rank $p=0,185$ e 0,737, respectivamente).

Para avaliar o impacto do longo intervalo de inclusão, dividimos os pacientes de acordo com a mediana de inclusão: 2005-2010 $(n=13)$ e 2011-2018 $(n=11)$ (Tabelas Suplementares 1 e 2). Apenas a dosagem de ureia apresentou diferença significativa entre os grupos 2005-2010 e 20112018 (67 [51-87] vs. 44 [38-49] mg/dL, respectivamente; $p=0,012)$. Não encontramos diferenças em relação às outras características basais, bem como em relação aos desfechos principais de mortalidade em 1 ano (15,4 vs. 18,2 \%, 
respectivamente; $\mathrm{p}=1,000)$ e taxas de novos sangramentos $(30,8$ vs. $18,2 \%$, respectivamente; $p=0,649)$. Houve uma tendência a menor indicação de tratamento clínico isolado no grupo 2011-2018 (46,2 vs. 9,1\%, respectivamente; $p=0,078$ ), porém sem significância estatística.

\section{Discussão}

Os principais achados do presente estudo são: 1) 50\% dos pacientes apresentaram sangramento gastrointestinal com necessidade de transfusão de hemáceas na admissão, demonstrando que a síndrome de Heyde é uma doença com potencial risco de morte; 2) não houve diferença nas taxas de ressangramento comparando pacientes submetidos ao tratamento intervencionista vs. tratamento clínico medicamentoso; 3) a sobrevida foi similar entre os grupos, inclusive no seguimento tardio; 4) o local de sangramento mais frequente foi o cólon ascendente.

A síndrome de Heyde é definida pela presença de estenose aórtica importante associada a sangramento gastrointestinal decorrente de angiodisplasias e, desde sua primeira descrição em 1958, sua fisiopatogênese não foi completamente elucidada. ${ }^{1}$ Vasos angiodisplásicos são as anormalidades vasculares mais comumente encontradas no trato gastrointestinal, e sua prevalência certamente aumenta com a idade, assim como a prevalência da estenose aórtica. ${ }^{2,4,11,13}$ Entretanto, a correlação entre estenose aórtica importante e a presença de lesões angiodisplásicas não parece ser casual. A obstrução da via de saída do ventrículo esquerdo reduz o fluxo gastrointestinal devido ao achatamento dos padrões de onda dos pulsos arteriais. Tal alteração levaria a isquemia esplâncnica relativa, favorecendo assim a neoangiogênese. ${ }^{4,14-17}$ Essa hipótese é corroborada por Greensteins et al., ${ }^{14}$ que estudaram a configuração da onda de pulso arterial na vasculatura mesentérica em pacientes com estenose aórtica, e por Stern et al., ${ }^{15}$ que avaliaram 33 pacientes com dispositivos de assistência ventricular não pulsátil e encontraram sangramentos decorrentes de angiodisplasias do intestino delgado em 13 deles. Além disso, a mucosa colônica isquêmica torna-se frágil, aumentado o risco de sangramento. Entretanto, o tratamento intervencionista da estenose aórtica não resolve as lesões angiodisplásicas, assim como documentado em três dos nossos casos, em que foi repetida a colonoscopia e/ou endoscopia digestiva alta após a intervenção valvar. ${ }^{2}$ Ademais, o cólon ascendente foi o local onde as lesões foram mais frequentemente encontradas, assim como descrito na literatura. ${ }^{12}$

Outro fator que contribui para o sangramento das lesões angiodisplásicas é a deficiência adquirida do vWF. Os vWF são glicoproteínas multiméricas que se ligam ao fator VIII, contribuindo para a formação do trombo plaquetário e agindo como um mediador da adesão plaquetária. ${ }^{2,3,6,18,19} \mathrm{Em}$ situações de estresse de cisalhamento decorrente da estenose valvar aórtica, o vWF altera sua estrutura, ocorrendo proteólise desses multímeros mediada pela enzima A Disintegrin And Metalloproteinase with Thrombospondin type 1 motif, member 13 (ADAMTS13). 2,3,6,18,19 Alguns autores sugerem que a intervenção valvar aórtica (cirúrgica ou transcateter) corrige tal deficiência do vWF, e drasticamente reduzindo a taxa de novos sangramentos. 5,6,11,12,19-21 Thompsom et al., ${ }^{12}$ em uma coorte de 34 anos, avaliaram 57 pacientes com síndrome de Heyde submetidos a troca valvar aórtica cirúrgica e demonstraram que $79 \%$ deles não apresentaram novos episódios de sangramento após a intervenção valvar após seguimento com mediana de 4,4 [0,1-15] anos. Os pacientes apresentavam características basais semelhantes às do nosso estudo, porém não havia grupo controle. Em nossa coorte de 13 anos e com mediana de seguimento de 2 [1-4,5] anos, 76,5\% dos pacientes também não apresentaram novos episódios de sangramento após a intervenção valvar aórtica. Entretanto, não houve diferença quando estes foram comparados aos pacientes mantidos em tratamento clínico medicamentoso, sugerindo que a intervenção teria o mesmo impacto que o tratamento clínico em relação a novos episódios hemorrágicos em pacientes com síndrome de Heyde.

A síndrome de Heyde não é descrita nas diretrizes atuais de tratamento das doenças valvares. Entretanto, devido à sugestão de resolução do sangramento após a correção da estenose aórtica, alguns autores advogam a indicação de intervenção valvar em pacientes com essa condição, independente da presença de sintomas ou complicadores, assim como realizado em dois pacientes do presente estudo. ${ }^{11,12,19-21}$ Um dado a favor da indicação de intervenção pela síndrome de Heyde é que esta pode ocasionar episódios com alto risco de mortalidade, assim como no estudo atual, em que metade dos pacientes necessitou de transfusão de concentrado de hemáceas na admissão. Em situações de anemia aguda, aqueles com estenose aórtica importante são incapazes de aumentar o débito cardíaco, devido à obstrução fixa da via de saída, sendo assim suscetíveis a eventos cardiovasculares, como insuficiência cardíaca e infarto do miocárdio tipo 2. Apesar disso, 29,2\% persistiram em tratamento clínico medicamentoso devido à escolha do paciente e do médico assistente. Pacientes do grupo clínico apresentavam uma tendência a serem mais idosos, porém sem outras diferenças estatisticamente significantes relacionadas às características basais. É importante salientar que tais pacientes foram acompanhados em um hospital terciário do sistema público de saúde brasileiro, no qual o TAVR não era uma opção terapêutica disponível no momento, justificando assim a alta taxa de tratamento conservador e os poucos casos de TAVR.

\section{Limitações}

O estudo atual sofre das limitações inerentes ao seu desenho. Um ponto importante é o tamanho da série que, apesar de relativamente pequena, até o presente momento é a maior série de casos de síndrome de Heyde da América Latina. Apesar disso, estamos sujeitos a erro tipo II para assegurar que o tratamento intervencionista não seja suficiente para a redução de eventos/novos sangramentos. Além disso, foi necessário longo tempo de inclusão, por se tratar de doença rara; todavia, a análise em relação ao tempo de inclusão não mostrou diferenças significativas. Outra limitação é o fato de que alguns aspectos, como fragilidade, pressão sistólica de artéria pulmonar, não puderam ser avaliados, devido às características do estudo, assim como não houve a avaliação da deficiência do vWF. Além disso, pacientes incluídos nos 
últimos anos tiveram menor seguimento, o que pode impactar a análise de sobrevida em longo prazo, apesar do ajuste estatístico pelo tempo de seguimento.

\section{Conclusão}

Em nossa coorte, pacientes com síndrome de Heyde tiveram alta taxa de sangramento com necessidade de transfusão sanguínea na admissão, sugerindo ser uma doença grave e com risco elevado de mortalidade. Não encontramos diferenças entre os grupos clínico e intervenção em relação a taxas de ressangramento e mortalidade tardia. Estudos prospectivos são necessários para confirmar se a intervenção valvar reduz a taxa de novos sangramentos e se a síndrome de Heyde deveria, por si só, ser indicativa de intervenção valvar. Entretanto, a raridade dessa entidade dificulta a realização de tais trabalhos.

\section{Contribuição dos autores}

Concepção e desenho da pesquisa: Rosa VEE, Fernandes JRC, Santis A, Spina GS, Sampaio RO, Tarasoutchi F; Obtenção

\section{Referências}

1. Heyde E. Gastrointestinal bleeding in aortic stenosis. The New England journal of medicine. 1958;259:196.

2. Warkentin TE, Moore JC, Anand SS, Lonn EM, Morgan DG. Gastrointestinal bleeding, angiodysplasia, cardiovascular disease, and acquired von willebrand syndrome. Transf Med Rev. 2003;17(4):272-86.

3. Morishima A, Marui A, Shimamoto T, Saji Y, Tambara K, Nishina T, Komeda M. Successful aortic valve replacement for heyde syndrome with confirmed hematologic recovery. Ann Thorac Surg. 2007;83(1):287-8.

4. Islam S, Cevik C, Islam E, Attaya H, Nugent K. Heyde's syndrome: A critical review of the literature. J Heart Valve Dis.2011;20(4):366-75.

5. Godino C, Lauretta L, Pavon AG, Mangieri A, Viani G, Chieffo A, etal. Heyde's syndrome incidence and outcome in patients undergoing transcatheter aortic valve implantation. J Am Coll Cardiol. 2013;61(6):687-9.

6. Loscalzo J. From clinical observation to mechanism—heyde's syndrome.N engl J Med. 2012;367(20):1954-6.

7. Baumgartner H, Falk V, Bax JJ, De Bonis M, Hamm C, Holm PJ, et al. 2017 esc/eacts guidelines for the management of valvular heart disease. Eu Heart J.2017;38(36):2739-91.

8. Nishimura RA, Otto CM, Bonow RO, Carabello BA, Erwin JP, Fleisher LA, et al. 2017 AHA/ACC focused update of the 2014 aha/acc guideline for the management of patients with valvular heart disease: A report of the american college of cardiology/american heart association task force on clinical practice guidelines.J Am Coll Cardiol. 2017;70(2):252-89.

9. Tarasoutchi F, Montera MW, Ramos AldO, Sampaio RO, Rosa VEE, Accorsi TAD, et al. Atualização das diretrizes brasileiras de valvopatias: Abordagem das lesões anatomicamente importantes. Arq Bras Cardiol. 2017;109(6 Suppl):1-34.

10. Tarasoutchi F, Montera M, Grinberg M, Barbosa M, Piñeiro D, Sánchez C, Barbosa M. Diretriz brasileira de valvopatias-sbc 2011/i diretriz interamericana de valvopatias-siac 2011.Arq Bras Cardiol. 2011;97(5 Suppl):01-67.

11. Natorska J, Mazur P, Undas A. Increased bleeding risk in patients with aortic valvular stenosis: From new mechanisms to new therapies. Thromb Res. 2016;139:85-9. de dados: Rosa VEE, Fernandes JRC, Santis A, Spina GS, Paixão MR, Pires JT, Bettega M, Accorsi TAD, Sampaio RO, Tarasoutchi F; Análise e interpretação dos dados: Rosa VEE, Ribeiro HB, Tarasoutchi F; Análise estatística: Rosa VEE, Ribeiro HB; Redação do manuscrito: Rosa VEE; Revisão crítica do manuscrito quanto ao conteúdo intelectual importante: Rosa VEE, Ribeiro HB, Fernandes JRC, Santis A, Spina GS, Paixão MR, Pires JT, Bettega M, Accorsi TAD, Sampaio RO, Tarasoutchi F.

\section{Potencial conflito de interesse}

Não há conflito com o presente artigo

\section{Fontes de financiamento}

O presente estudo não teve fontes de financiamento externas.

\section{Vinculação acadêmica}

Não há vinculação deste estudo a programas de pós-graduação.

12. Thompson JL, Schaff HV, Dearani JA, Park SJ, Sundt TM, Suri RM, etal. Risk of recurrent gastrointestinal bleeding after aortic valve replacement in patients with heyde syndrome. J Thorac Cardiovasc Surg. 2012;144(1):112-6.

13. Pate GE, Mulligan A. An epidemiological study of heyde's syndrome: An association between aortic stenosis and gastrointestinal bleeding.J Heart Valve Dis. 2004;13(5):713-6.

14. Greenstein RJ, McElhinney AJ, Reuben D, Greenstein AJ. Colonic vascular ectasias and aortic stenosis: Coincidence or causal relationship? Am J Surg. 1986;151(3):347-51.

15. Stern DR, Kazam J, Edwards P, Maybaum S, Bello RA, D'Alessandro DA, et al. Increased incidence of gastrointestinal bleeding following implantation of the heartmate ii lvad. J Card Surg. 10;25(3):352-6.

16. Letsou GV, Shah N, Gregoric ID, Myers TJ, Delgado R, Frazier O. Gastrointestinal bleeding from arteriovenous malformations in patients supported by the jarvik 2000 axial-flow left ventricular assist device. Heart Lung Transpl. 2005;24(1):105-9.

17. Nishimura T, Tatsumi E, Takaichi S, Taenaka Y, Wakisaka Y, Nakatani T, et al. Morphologic changes of the aortic wall due to reduced systemic pulse pressure in prolonged non pulsatile left heart bypass. ASAIO J. 1997;43(5):M691-5.

18. Ledingham D. Heyde's syndrome: Exploring the link between aortic stenosis and an acquired bleeding disorder. BMJ case reports. 2013;2013:bcr2013009306

19. Spangenberg T, Budde U, Schewel D, Frerker C, Thielsen T, Kuck K-H, Schäfer $U$. Treatment of acquired von willebrand syndrome in aortic stenosis with transcatheter aortic valve replacement. JACC: CardiovascIntervent. 2015;8(5):692-700.

20. Jackson CS, Gerson LB. Management of gastrointestinal angiodysplastic lesions (giads): A systematic review and meta-analysis. Am J Gastroenterol. 2014;109(4):474-83.

21. King RM, Pluth JR, Giuliani ER. The association of unexplained gastrointestinal bleeding with calcific aortic stenosis. Ann Thorac Surg. 1987;44(5): 514-6. 